\title{
Non-Vitamin K Antagonist Oral Anticoagulants and the Gastrointestinal Bleeding Risk in Real-World Studies
}

\author{
Larisa Anghel 1,2, Radu Sascău 1,2,*, Anca Trifan 1,3 (D), Ioana Mădălina Zota 1,2 $\mathbb{D}$ \\ and Cristian Stătescu 1,2 (D) \\ 1 Internal Medicine Department, “Grigore T. Popa” University of Medicine and Pharmacy, 700503 Iași, \\ Romania; larisa.anghel@umfiasi.ro (L.A.); ancatrifan@yahoo.com (A.T.); \\ madalina.chiorescu@gmail.com (I.M.Z.); cstatescu@gmail.com (C.S.) \\ 2 Cardiology Department, Cardiovascular Diseases Institute, "Prof. Dr. George I.M. Georgescu", \\ 700503 Iași, Romania \\ 3 Gastroenterology and Hepatology Department, Gastroenterology and Hepatology Institute, \\ 700019 Iași, Romania \\ * Correspondence: radu.sascau@gmail.com; Tel.: +40-0232-211834
}

Received: 26 March 2020; Accepted: 7 May 2020; Published: 9 May 2020

\begin{abstract}
In the present study, we aimed to provide evidence from high-quality real world studies for a comprehensive and rigorous analysis on the gastrointestinal bleeding (GIB) risk for non-vitamin $\mathrm{K}$ antagonist oral anticoagulants (NOACs). We performed a systematic search of MEDLINE, EMBASE and PUBMED, and of 286 records screened, we included data from 11 high-quality real-world studies, coordinated by independent research groups over the last 3 years, that reported major GIB events in patients given NOACs or vitamin K antagonists for patients with nonvalvular atrial fibrillation. The lowest risk of gastrointestinal bleeding was with apixaban compared with warfarin (hazard ratio (HR) for GIB for apixaban ranging between 0.45 (95\% confidence interval (CI) 0.34 to 0.59 ) and 1.13 (95\% CI 0.79 to 1.63)). Apixaban was associated with a lower risk of GI bleeding than dabigatran ((HR ranging between 0.39 (95\% CI 0.27 to 0.58$)$ and 0.95 (95\% CI 0.65 to 1.18$)$ ) or rivaroxaban ((HR ranging between 0.33 (95\% CI 0.22 to 0.49$)$ and 0.82 (95\% CI 0.62 to 1.08$)$ ). The results of our study confirm a low or a similar risk for major GIB between patients receiving apixaban or dabigatran compared with warfarin, and apixaban appears to be associated with the lowest risk of GIB.
\end{abstract}

Keywords: non-vitamin K antagonist oral anticoagulants; gastrointestinal bleeding; safety profile; real-world studies

\section{Introduction}

Since warfarin was approved for use in 1954, it has been the mainstay of anticoagulant treatment for patients with atrial fibrillation, deep vein thrombosis and pulmonary embolism. However, the use of vitamin K antagonists (VKAs) has many limitations, such as the need to ensure proper anticoagulation control by regular monitoring and also many interactions with drugs and diet. As a result, 30-50\% of patients are undertreated [1-4]. In the last 10 years, the US Food and Drug Administration (FDA) has approved non-vitamin $\mathrm{K}$ antagonist oral anticoagulants (NOACs), long-awaited alternatives to the vitamin $\mathrm{K}$ antagonists that offer relative efficacy, safety and convenience. This new class of drugs includes dabigatran, rivaroxaban, apixaban and edoxaban. They have the convenience of fixed dosing with no need for laboratory monitoring or dietary discretion [5-11]. But despite these apparent advantages, debate still remains regarding the gastrointestinal bleeding (GIB) risk [12-18]. 
Compared with vitamin K antagonists or heparins, NOACs have rapid onset and offset of action, predictable pharmacodynamics, fewer food-drug and drug-drug interactions and can reversibly and directly inhibit a specific factor of the coagulation cascade [19-23] (Table 1).

Table 1. Indications and dosing of NOACs in Europe.

\begin{tabular}{|c|c|c|c|}
\hline Indication & Dabigatran & Apixaban & Rivaroxaban \\
\hline \multirow{3}{*}{$\begin{array}{l}\text { Non-valvular atrial } \\
\text { fibrillation }\end{array}$} & $150 \mathrm{mg}$ b.i.d & $5 \mathrm{mg}$ b.i.d & $20 \mathrm{mg}$ daily \\
\hline & $\begin{array}{l}110 \mathrm{mg} \text { b.i.d if age } \geq 80 \text { years } \\
\text { (may consider } 110 \mathrm{mg} \text { b.i.d } \\
\text { also if increased risk of } \\
\text { bleeding) }\end{array}$ & $\begin{array}{c}2.5 \mathrm{mg} \text { b.i.d if } \mathrm{CrCl} \\
15-29 \mathrm{~mL} / \mathrm{min} \text { OR two out of } \\
\text { the following: } \\
\text { age } \geq 80 \text { years, } \mathrm{BW} \leq 60 \mathrm{~kg} \\
\mathrm{Cr} \geq 1.5 \mathrm{mg} / \mathrm{dL}\end{array}$ & - \\
\hline & Avoid if $\mathrm{CrCl}<30 \mathrm{~mL} / \mathrm{min}$ & Avoid if $\mathrm{CrCl}<15 \mathrm{~mL} / \mathrm{min}$ & Avoid if $\mathrm{CrCl}<15 \mathrm{~mL} / \mathrm{min}$ \\
\hline \multirow{3}{*}{$\begin{array}{l}\text { Treatment and } \\
\text { prevention of recurrent } \\
\text { deep vein thrombosis/ } \\
\text { pulmonary embolism }\end{array}$} & $\begin{array}{l}150 \mathrm{mg} \text { b.i.d after } 5 \text { days of } \\
\text { initial therapy with a } \\
\text { parenteral anticoagulant }\end{array}$ & $\begin{array}{l}10 \mathrm{mg} \text { b.i.d for } 1 \text { week, then } \\
5 \mathrm{mg} \text { b.i.d }\end{array}$ & $\begin{array}{l}15 \mathrm{mg} \text { b.i.d for } 3 \text { weeks, then } \\
20 \mathrm{mg} \text { daily }\end{array}$ \\
\hline & $\begin{array}{l}110 \mathrm{mg} \text { b.i.d after } 5 \text { days of } \\
\text { initial therapy with a } \\
\text { parenteral anticoagulant if } \\
\text { age } \geq 80 \text { years (may consider } \\
110 \mathrm{mg} \text { b.i.d also if increased } \\
\text { risk of bleeding) }\end{array}$ & - & - \\
\hline & Avoid if $\mathrm{CrCl}<30 \mathrm{~mL} / \mathrm{min}$ & Avoid if $\mathrm{CrCl}<15 \mathrm{~mL} / \mathrm{min}$ & Avoid if $\mathrm{CrCl}<15 \mathrm{~mL} / \mathrm{min}$ \\
\hline
\end{tabular}

NOACs: non-vitamin $\mathrm{K}$ antagonist oral anticoagulants; $\mathrm{CrCl}$ : Creatinine clearance; BW: Body weight; Cr: Creatinine; b.i.d: Twice a day.

Dabigatran targets thrombin and all xabans (apixaban, rivaroxaban, edoxaban) target factor Xa. The beginning of the anticoagulation effect is rapid and begins two hours following the first dose and is lost within $24 \mathrm{~h}$ after discontinuation of the drugs. Direct anti-Xa inhibitors are able to inhibit both free and prothrombinase-bound factor Xa, and may also be able to inhibit clot-associated factor Xa. It is important to mention that rivaroxaban does not react with antibodies implicated in heparin-induced thrombocytopenia $[21,24,25]$.

Dabigatran is a direct thrombin inhibitor, administered as a prodrug (dabigatran etexilate) and absorbed principally in the stomach and proximal small bowel as an inactive prodrug. Serum and hepatic esterases metabolize this inactive prodrug to the active drug. The bioavailability of the drug is low, approximately 3-7\%, with the unabsorbed dabigatran being converted to active dabigatran in the distal bowel and then excreted in the feces [25-28]. The majority of non-absorbed drug is excreted in the stool and the absorbed drug is mainly excreted unchanged by the kidneys. Compared with warfarin, which is not activated in the bowel, the active dabigatran in the distal bowel may promote gastrointestinal bleeding $[25,26]$. There are also studies reporting that dabigatran is associated with esophagitis and gastric ulceration, suggesting that the drug may directly injure the gastrointestinal mucosa [29]. Dabigatran may be given at a dose of $150 \mathrm{mg}$ b.i.d or $110 \mathrm{mg}$ b.i.d. The dose of $75 \mathrm{mg}$ b.i.d can be administered in the presence of renal insufficiency (creatinine clearance $(\mathrm{CrCl})<50 \mathrm{~mL} / \mathrm{min}$ ). The drug is contraindicated in patients with severe renal impairment $(\mathrm{CrCl}<30 \mathrm{~mL} / \mathrm{min})$ or advanced liver disease [30-34].

Apixaban is a direct inhibitor of factor Xa and has a bioavailability of $50 \%$. Almost $25 \%$ of the absorbed drug is excreted by the kidney with a half-life of around $12 \mathrm{~h}$. Apixaban is administered at a dose of $5 \mathrm{mg}$ b.i.d, and $2.5 \mathrm{mg}$ b.i.d if patients have at least 2 of the following features: age 80 years or older, body weight $60 \mathrm{~kg}$ or less, or serum creatinine $1.5 \mathrm{mg} / \mathrm{dL}$ or more [35-40]. 
Rivaroxaban is also a direct factor Xa inhbitior, with a bioavailability of $66 \%$ and a half-life that ranges from 6 to $13 \mathrm{~h}[35,41]$. One-third of the absorbed drug is excreted by the kidney and two-thirds is metabolized by the liver into inactive forms. Rivaroxaban is administered at a dose of $20 \mathrm{mg}$ daily, and $15 \mathrm{mg}$ daily if the $\mathrm{CrCl}$ is $<50 \mathrm{~mL} / \mathrm{min}$. The drug is also contraindicated in severe renal impairment $(\mathrm{CrCl}<15 \mathrm{~mL} / \mathrm{min})$ and advanced liver disease [41-45].

The anticoagulant effect can be local and/or systematic and the sites of gastrointestinal bleeding differ for individual NOACs. They have a systemic anticoagulant effect and also a local effect such as: incomplete absorption (topical anticoagulant effect), direct caustic effect (tartaric acid in dabigatran) or inhibition of mucosal healing [24]. When compared with warfarin, aspirin or non-steroidal anti-inflammatory drugs, where upper gastrointestinal bleeding predominates [46], in the RE-LY trial, lower GIB was found in $53 \%$ of dabigatran users. In this post hoc analysis of the Randomized Evaluation of Long-Term Anticoagulation Therapy (RE-LY) trial, comparing dabigatran to warfarin, Kolb et al. [24] reviewed the cases of suspected GI bleeding. They collected data on the causative lesions and the site and acuity of bleeding within the bowel. They localized the bleeding in approximately two-thirds of the cases, and $47 \%$ of them were detected in the upper GI tract and 39\% in the colon. It was interesting that the rate of bleeding in the upper GI tract was similar between the two doses of dabigatran studied (110 and $150 \mathrm{mg}$ ) as compared with warfarin, whereas lower GI bleeding (which included colonic, jejunal and ileal sources) was more frequent in patients treated with dabigatran as compared with warfarin (relative risk was 1.78 for dabigatran $110 \mathrm{mg}$ and 2.23 for dabigatran $150 \mathrm{mg}$ ). This probably is related to the incomplete absorption of the active NOACs in the upper GI tract, which leads to an increased availability of dabigatran in the lower GI tract and also a topical effect on the mucosa leading to bleeding. This gastrointestinal bleeding risk is higher especially in the presence of preexisting lesions like angiodysplasias and erosions [47-50]. A recent study published by Contaldo et al. evaluated the gastrointestinal bleeding caused by NOACs with videocapsule endoscopy. They enrolled 109 patients with iron deficiency anaemia, 18 of them taking oral anticoagulants, and demonstrated a trend in the association of oral anticoagulant use with small intestinal lesions at videocapsule endoscopy, despite a non-statistical significance (odds ratio $(\mathrm{OR})=3.38 ; 95 \%$ confidence interval $(\mathrm{CI}) 0.73-15.70 ; P=0.10$ ). This study highlights the usefulness of videocapsule endoscopy in providing clear information in patients with unexplained iron deficiency anaemia [51].

Even if apixaban and rivaroxaban are both factor Xa inhibitors, with similar bioavailability, and are administered in active form, the risk of GIB differs in these two agents, and this may be related to the higher peak level of once-daily dosing of rivaroxaban than the twice-daily dosing of apixaban $[52,53]$. The risk factors for NOACs-related gastrointestinal bleeding are summarized in Table 2.

Table 2. Factors associated with NOACs-related gastrointestinal bleeding.

\begin{tabular}{l}
\hline \multicolumn{1}{c}{ Risk Factors } \\
\hline 1. Higher dose of dabigatran: a dose of $150 \mathrm{mg}$ b.i.d \\
2. Concomitant use of ulcerogenic agents like antiplatelet agents, non-steroidal anti-inflammatory drugs or steroid \\
3. Older age: $\geq 75$ years \\
4. Renal impairment with a creatinine clearance $<50 \mathrm{~mL} / \mathrm{min}$ \\
5. Prior history of peptic ulcers or GIB \\
6. Helicobacter pylori infection \\
7. Pre-existing GI tract lesions such as: diverticulosis, angiodysplasias \\
8. Ethnicity: western population \\
9. HAS-BLED score $\geq 3$
\end{tabular}

Protective Factors

Gastroprotective agents: proton pump inhibitors or histamine H2-receptor antagonists

HAS-BLED: a scoring system developed to assess 1-year risk of major bleeding in patients taking anticoagulants with atrial fibrillation. The score is between 0 and 9 , based on seven parameters: hypertension, abnormal renal/liver function (1 point each), stroke, bleeding history or predisposition, labile INR, elderly ( $>65$ years), drug/alcohol concomitantly (1 point each). 
Considering the fact that debate still remains regarding the gastrointestinal bleeding risk for patients on anticoagulant therapy, either warfarin or NOACs, we aimed to highlight evidence from high-quality real world studies regarding the GIB risk for oral anticoagulants.

\section{Experimental Section}

The availability of warfarin and these NOACs in real-world clinical practice allows opportunities for comparative effectiveness analyses, particularly of the gastrointestinal bleeding risk of these drugs when used outside the controlled setting of clinical trials. Because edoxaban was recently approved by the FDA in January 2015 and introduced to the market and because little real-world data are available, this study only focused on warfarin, dabigatran, rivaroxaban and apixaban. The main objective of our study was to compare the gastrointestinal bleeding risk among anticoagulated non-valvular atrial fibrillation patients on warfarin, dabigatran, rivaroxaban and apixaban. We compared GIB risk between each NOAC and warfarin, but also a direct pairwise comparison between individual NOACs.

Real-world studies (RWSs), by integrating data from electronic health records, claims databases and disease registries, could extend findings of RCTs to large patient populations in real-world practice. The idealized settings of a clinical trial may not adequately reflect the real-world safety profile of NOACs as they are prescribed in routine clinical practice [54]. Therefore, RWSs are needed to clarify which anticoagulant would be the best choice for atrial fibrillation patients, to assess specifically the gastrointestinal safety profile.

In the present study, we summarized evidence from high-quality RWSs for a comprehensive and rigorous analysis on the GIB risk for NOACs. We followed the PRISMA (preferred reporting items for systematic reviews and meta-analyses) guidelines when performing this research. These studies were selected by performing a systematic search of MEDLINE, EMBASE and PUBMED, using the following items: gastrointestinal bleeding risk, GIB, dabigatran, rivaroxaban, apixaban, warfarin, real-world studies, atrial fibrillation. We included in the research only high-quality real-world studies that fulfilled the following criteria: (1) reported major gastrointestinal bleeding events in patients given NOACs or warfarin; (2) available data on clinical events; (3) adjusted hazard ratios between each NOAC versus warfarin and from direct pairwise comparison of different NOACs for major gastrointestinal bleeding; (4) studies coordinated by independent research groups, published between 01 January 2017 and 31 December 2019. Considering that funding bias may be a form of publication bias, a phenomenon that is also recognized and studied by the researchers, we preferred to include only real-world data that were not sponsored by pharmaceutical companies. We excluded (1) animal-based studies; (2) non-English-based studies; (3) abstract, editorials, case reports and reviews (Figure 1). 


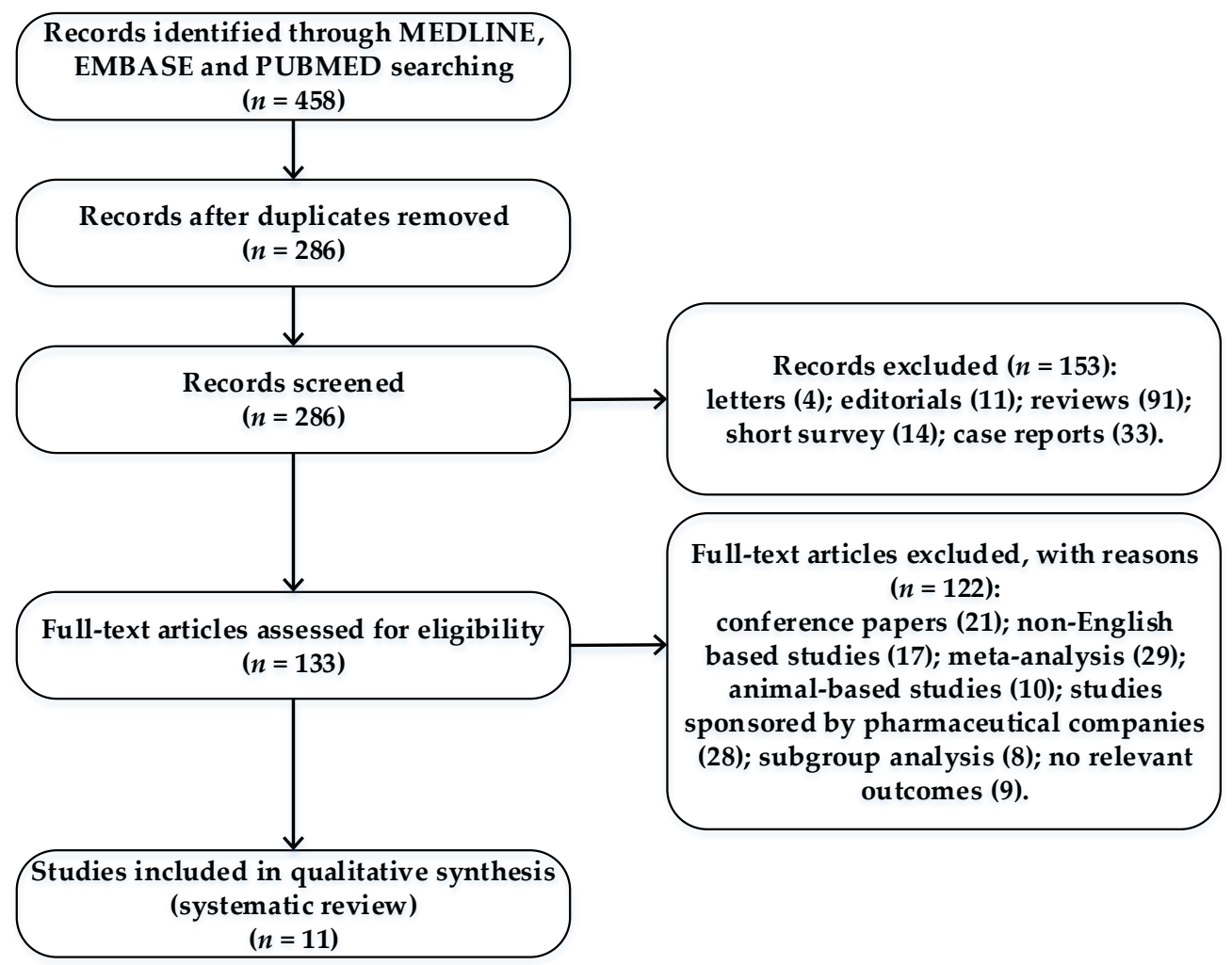

Figure 1. Flow chart with the process of article selection.

Of 286 records screened, we included data from 11 high-quality real-world studies that reported major gastrointestinal bleeding events in patients given NOACs or vitamin K antagonists (Table 3). 
Table 3. Real-world studies that analyzed NOACs-related gastrointestinal bleeding.

\begin{tabular}{|c|c|c|c|c|c|c|}
\hline Study & Country & Obser-Vation Period & $\begin{array}{l}\text { Oral Anticoagu-Lants } \\
\text { Analyzed }\end{array}$ & Methodology & Population & Endpoint \\
\hline Abraham et al. [54] & USA & $\begin{array}{l}\text { October } 2010 \text { to } \\
\text { February } 2015\end{array}$ & $\begin{array}{l}\text { Apixaban, dabigatran or } \\
\text { rivaroxaban }\end{array}$ & $\begin{array}{c}\text { Retrospective cohort study based } \\
\text { on medical and pharmacy claims } \\
\text { data from OptumLabs Data } \\
\text { Warehouse using PSM }\end{array}$ & $\begin{array}{l}\text { 43,303 adults diagnosed with } \\
\text { NVAF who had an } \\
\text { indexprescription for apixaban, } \\
\text { dabigatran, or rivaroxaban }\end{array}$ & Bleeding \\
\hline Adeboyeje et al. [55] & USA & $\begin{array}{l}\text { November } 2010 \text { to } \\
\text { February } 2015\end{array}$ & $\begin{array}{l}\text { Apixaban, dabigatran, } \\
\text { rivaroxaban, warfarin }\end{array}$ & $\begin{array}{l}\text { Retrospective cohort study based } \\
\text { on data from a commercially } \\
\text { insured population in the U.S. }\end{array}$ & $\begin{array}{c}\text { 44,057 patients diagnosed with } \\
\text { NAVF, who used warfarin }(23,431), \\
\text { dabigatran (8539), apixaban }(3689) \\
\text { and rivaroxaban }(8398)\end{array}$ & Bleeding \\
\hline Andersson et al. [30] & Denmark & $\begin{array}{l}\text { July } 2013 \text { to March } \\
2016\end{array}$ & $\begin{array}{l}\text { Apixaban, dabigatran or } \\
\text { rivaroxaban }\end{array}$ & $\begin{array}{l}\text { Retrospective cohort study based } \\
\text { on nationwide registers }\end{array}$ & $\begin{array}{l}\text { 12,638 NVAF patients, propensity } \\
\text { scores in a 1:1 ration comparing } \\
\text { (apixaban vs. dabigatran }=6470 ; \\
\text { apixaban vs. rivaroxaban }=7352 ; \\
\text { rivaroxaban vs. dabigatran }= \\
5440 \text { patients) }\end{array}$ & $\begin{array}{l}\text { Effective-ness + } \\
\text { bleeding }\end{array}$ \\
\hline Forslund et al. [56] & Sweden & $\begin{array}{l}\text { January } 2012 \text { to } \\
\text { December } 2015\end{array}$ & $\begin{array}{l}\text { Apixaban, dabigatran, } \\
\text { rivaroxaban, warfarin }\end{array}$ & $\begin{array}{l}\text { Population-based retrospective } \\
\text { cohort study based on data from } \\
\text { the Stockholm Region } \\
\text { administrative health data } \\
\text { register containing healthcare } \\
\text { utilization and prescription data }\end{array}$ & $\begin{array}{l}\text { 22,198 adults with NVAF who were } \\
\text { naive for either warfarin or one of } \\
\text { the following NOACs: apixaban, } \\
\text { dabigatran, or rivaroxaban during } \\
\text { the study period. }\end{array}$ & $\begin{array}{l}\text { Effective-ness + } \\
\text { bleeding }\end{array}$ \\
\hline Graham et al. [57] & USA & $\begin{array}{l}\text { October } 2010 \text { to } \\
\text { September } 2015\end{array}$ & $\begin{array}{l}\text { Apixaban, dabigatran, } \\
\text { rivaroxaban, warfarin }\end{array}$ & $\begin{array}{l}\text { Retrospective new-users cohort } \\
\text { study based on data from } \\
\text { Medicare beneficiaries }\end{array}$ & $\begin{array}{c}\text { 183,318 warfarin, } 86,198 \\
\text { dabigatran, } 106,389 \text { rivaroxaban } \\
\text { and } 73,039 \text { apixaban users }\end{array}$ & $\begin{array}{l}\text { Effective-ness + } \\
\text { bleeding }\end{array}$ \\
\hline Hernandez et al. [58] & USA & $\begin{array}{l}\text { January } 2013 \text { to } \\
\text { December } 2014\end{array}$ & $\begin{array}{l}\text { Apixaban, dabigatran, } \\
\text { rivaroxaban, warfarin }\end{array}$ & $\begin{array}{l}\text { Retrospective database analysis } \\
\text { from a random sample of } \\
\text { Medicare beneficiaries }\end{array}$ & $\begin{array}{l}\text { 41,366 adults with NVAF who were } \\
\text { naive for either warfarin or one of } \\
\text { the following NOACs: apixaban, } \\
\text { dabigatran or rivaroxaban during } \\
\text { the study period. }\end{array}$ & $\begin{array}{l}\text { Effective-ness + } \\
\text { bleeding }\end{array}$ \\
\hline Larsen et al. [59] & Denmark & $\begin{array}{l}\text { August } 2011 \text { to } \\
\text { October } 2015\end{array}$ & $\begin{array}{l}\text { Warfarin, apixaban, } \\
\text { dabigatran, rivaroxaban } \\
\text { (only standard dose) }\end{array}$ & $\begin{array}{l}\text { Retrospective database analysis } \\
\text { using propensity score weighting } \\
\text { (inverse probability of treatment } \\
\text { weighting) }\end{array}$ & $\begin{array}{l}\text { 61,678 NVAF patients, naive to } \\
\text { OAC, first time on DOAC } \\
\text { or warfarin }\end{array}$ & $\begin{array}{l}\text { Effective-ness + } \\
\text { bleeding }\end{array}$ \\
\hline Nielsen et al. [60] & Denmark & $\begin{array}{l}\text { August } 2011 \text { to } \\
\text { February } 2016\end{array}$ & $\begin{array}{l}\text { Warfarin, apixaban, } \\
\text { dabigatran, rivaroxaban } \\
\text { (only reduced dose) }\end{array}$ & $\begin{array}{c}\text { Retrospective analysis using } \\
\text { propensity score weighting } \\
\text { (inverse probability of treatment } \\
\text { weighting) }\end{array}$ & $\begin{array}{l}\text { 55,644 NVAF patients, naïve to } \\
\text { OAC, first time on DOAC or } \\
\text { warfarin, all restricted to } \\
\text { reduced dose }\end{array}$ & $\begin{array}{l}\text { Effective-ness + } \\
\text { bleeding }\end{array}$ \\
\hline
\end{tabular}


Table 3. Cont.

\begin{tabular}{|c|c|c|c|c|c|c|}
\hline Study & Country & Obser-Vation Period & $\begin{array}{c}\text { Oral Anticoagu-Lants } \\
\text { Analyzed }\end{array}$ & Methodology & Population & Endpoint \\
\hline Noseworthy et al. [61] & USA & $\begin{array}{l}\text { October } 2010 \text { to June } \\
2015\end{array}$ & $\begin{array}{c}\text { Rivaroxaban, dabigatran, } \\
\text { apixaban }\end{array}$ & $\begin{array}{c}\text { Retrospective analysis using } \\
\text { administrative claims, using } \\
\text { PSM and Cox proportional } \\
\text { hazards regression }\end{array}$ & $\begin{array}{l}\text { 57,788 NVAF patients, including } \\
\text { patients with prior warfarin } \\
\text { exposure. Apixaban used as } \\
\text { reference category }\end{array}$ & $\begin{array}{l}\text { Effective-ness }+ \\
\text { bleeding }\end{array}$ \\
\hline Vinogradova et al. [62] & UK & $\begin{array}{l}\text { January } 2011 \text { to } \\
\text { October } 2016\end{array}$ & $\begin{array}{c}\text { Warfarin, apixaban, } \\
\text { dabigatran, rivaroxaban }\end{array}$ & $\begin{array}{l}\text { Prospective open cohort study } \\
\text { using two primary care } \\
\text { databases representative of the } \\
\text { national population }\end{array}$ & $\begin{array}{c}\text { 132,231 warfarin, } 7744 \text { dabigatran, } \\
37,863 \text { rivaroxaban and } 18,223 \\
\text { apixaban users, subgrouped into } \\
103,270 \text { patients with atrial } \\
\text { fibrillation and } 92,791 \text { without } \\
\text { atrial fibrillation }\end{array}$ & Bleeding \\
\hline Yao et al. [63] & USA & $\begin{array}{l}\text { October } 2010 \text { to } \\
\text { June } 2015\end{array}$ & $\begin{array}{c}\text { Warfarin, apixaban, } \\
\text { dabigatran, rivaroxaban }\end{array}$ & $\begin{array}{c}\text { Retrospective database analyses } \\
\text { using PSM }\end{array}$ & $\begin{array}{l}\text { 125,243 NVAF patients, three } 1: 1 \\
\text { PSM cohorts (apixaban = } 15,390 \\
\text { dabigatran }=28,614 ; \\
\text { rivaroxaban }=32,350)\end{array}$ & $\begin{array}{l}\text { Effective-ness + } \\
\text { bleeding }\end{array}$ \\
\hline
\end{tabular}

NOACs: non-vitamin K antagonist oral anticoagulants; NVAF: nonvalvular atrial fibrillation; OAC: oral anticoagulant; DOAC: direct oral anticoagulant; PSM: propensity score matching. 


\section{Results}

\subsection{Characteristics of Patients Included in the Studies}

The primary outcome was major gastrointestinal bleeding, according to International Society on Thrombosis and Hemostasis criteria [64]. Bleeding was detected by upper endoscopy or colonoscopy, depending on the patient's symptoms and personal pathological background. Data extracted from these studies included baseline characteristics, patient demographics, co-morbidities, CHA2DS2-VASC score, HAS-BLED score, pharmacologic risk factors (antiplatelet agents, non-steroidal anti-inflammatory drugs) for gastrointestinal bleeding and also data on major GI bleeding for patients with NOACs and vitamin $\mathrm{K}$ antagonists. After propensity-score matching, cohorts were closely balanced for all covariates, standardized differences of all baseline characteristics were $<10 \%$ in all the studies, demonstrating similarity of comparators with regard to the important socio-demographic, co-morbidity and pharmacological risk factors (Table 4).

Table 4. Baseline characteristics of patients included in the studies.

\begin{tabular}{|c|c|c|c|c|c|}
\hline & Dabigatran & Rivaroxaban & Apixaban & Warfarin & $\begin{array}{l}\text { Maximum } \\
\text { SMD }\end{array}$ \\
\hline \multicolumn{6}{|l|}{ Study Abraham et al. [54] } \\
\hline Age group (mean) & $68.8(11.4)$ & $70.6(11.4)$ & $72.3(11.1)$ & NA & - \\
\hline Male & $60.5 \%$ & $57.1 \%$ & $54 \%$ & NA & - \\
\hline CHA2DS2-VASC (mean) & $3.6(1.9)$ & $3.8(1.9)$ & $4.0(1.9)$ & NA & - \\
\hline HAS-BLED (mean) & $2.2(1.2)$ & $2.4(1.2)$ & $2.4(1.2)$ & NA & - \\
\hline $\begin{array}{l}\text { Antiplatelet or non-steroidal } \\
\text { anti-inflammatory drugs }\end{array}$ & $10.7 \%$ & $12.1 \%$ & $12.3 \%$ & NA & - \\
\hline \multicolumn{6}{|l|}{ Study Adeboyeje et al. [55] } \\
\hline Age group (mean) & $70(12.3)$ & $70(12.3)$ & 70 (12.6) & $70(12.2)$ & 0.01 \\
\hline Male & $58.9 \%$ & $58.7 \%$ & $59.5 \%$ & $59.1 \%$ & 0.01 \\
\hline CHA2DS2-VASC (mean) & $3.3(1.9)$ & $3.3(1.9)$ & $3.3(1.9)$ & $3.3(1.8)$ & 0.01 \\
\hline HAS-BLED (mean) & $2.1(1.4)$ & $2.1(1.4)$ & $2.1(1.4)$ & $2.1(1.4)$ & 0.01 \\
\hline $\begin{array}{l}\text { Antiplatelet or non-steroidal } \\
\text { anti-inflammatory drugs }\end{array}$ & $19.9 \%$ & $20.5 \%$ & $20.2 \%$ & $20.2 \%$ & 0.01 \\
\hline \multicolumn{6}{|l|}{ Study Andersson et al. [30] } \\
\hline Age group (mean) & $65.7(7.3)$ & $72.0(9.8)$ & $71.9(9.1)$ & NA & - \\
\hline Male & $64 \%$ & $56 \%$ & $63 \%$ & NA & - \\
\hline CHA2DS2-VASC & - & - & - & NA & - \\
\hline HAS-BLED & - & - & - & NA & - \\
\hline $\begin{array}{c}\text { Antiplatelet or non-steroidal } \\
\text { anti-inflammatory drugs }\end{array}$ & $59.8 \%$ & $61.8 \%$ & $57.8 \%$ & NA & - \\
\hline \multicolumn{6}{|l|}{ Study Forslund et al. [56] } \\
\hline Age group (mean) & $69.9(11.3)$ & $74.0(10.3)$ & $75.0(10.8)$ & $74.1(11.0)$ & - \\
\hline Male & $60 \%$ & $54.6 \%$ & $54.6 \%$ & $55.4 \%$ & - \\
\hline CHA2DS2-VASC (mean) & 3. $01(1.89)$ & $3.59(1.88)$ & $3.69(1.9)$ & $3.68(1.91)$ & - \\
\hline HAS-BLED & - & - & - & - & - \\
\hline Antiplatelet drugs & $46.73 \%$ & $55.74 \%$ & $47.73 \%$ & $54.96 \%$ & - \\
\hline
\end{tabular}


Table 4. Cont.

\begin{tabular}{|c|c|c|c|c|c|}
\hline & Dabigatran & Rivaroxaban & Apixaban & Warfarin & $\begin{array}{l}\text { Maximum } \\
\text { SMD }\end{array}$ \\
\hline \multicolumn{6}{|l|}{ Study Graham et al. [57] } \\
\hline Age group (mean) & 75.5 & 74.9 & 75.2 & 75.8 & 0.15 \\
\hline Male & $52.4 \%$ & $53.9 \%$ & $52.2 \%$ & $52 \%$ & 0.04 \\
\hline CHA2DS2-VASC $(\geq 2)$ & $96.7 \%$ & $96.6 \%$ & $97.4 \%$ & $97.1 \%$ & 0.04 \\
\hline HAS-BLED $(\geq 3)$ & $44.7 \%$ & $43.7 \%$ & $47.8 \%$ & $45.8 \%$ & 0.03 \\
\hline $\begin{array}{l}\text { Antiplatelet or non-steroidal } \\
\text { anti-inflammatory drugs }\end{array}$ & $28.3 \%$ & $28.2 \%$ & $29.5 \%$ & $27.2 \%$ & 0.04 \\
\hline \multicolumn{6}{|l|}{ Study Hernandez et al. [58] } \\
\hline Age group (mean) & $74.9(8.7)$ & $76.4(8.6)$ & $77.4(8.6)$ & $76.0(10.3)$ & $<0.001$ \\
\hline Male & $47.0 \%$ & $43.7 \%$ & $42.5 \%$ & $43.1 \%$ & $<0.001$ \\
\hline CHA2DS2-VASC (mean) & $4.26(1.74)$ & $4.55(1.78)$ & $4.68(1.73)$ & $4.8(1.82)$ & $<0.001$ \\
\hline HAS-BLED (mean) & $3.49(0.93)$ & $3.65(0.95)$ & $3.71(0.93)$ & $3.71(1.0)$ & $<0.001$ \\
\hline $\begin{array}{l}\text { Antiplatelet or non-steroidal } \\
\text { anti-inflammatory drugs }\end{array}$ & $22.5 \%$ & $25.2 \%$ & $25.0 \%$ & $21.3 \%$ & $<0.001$ \\
\hline \multicolumn{6}{|l|}{ Study Larsen et al. [59] } \\
\hline Age group (mean) & $67.6(5.6)$ & $71.8(7.1)$ & $71.3(5.9)$ & $72.4(7.4)$ & 0.02 \\
\hline Male & $66.1 \%$ & $57.9 \%$ & $60.3 \%$ & $58.8 \%$ & 0.02 \\
\hline CHA2DS2-VASC (mean) & $2.2(1.4)$ & $2.8(1.6)$ & $2.8(1.6)$ & $2.8(1.7)$ & 0.02 \\
\hline HAS-BLED (mean) & $2.0(1.1)$ & $2.2(1.2)$ & $2.3(1.2)$ & $2.2(1.2)$ & 0.01 \\
\hline $\begin{array}{l}\text { Aspirin or non-steroidal } \\
\text { anti-inflammatory drugs }\end{array}$ & $62.7 \%$ & $60.4 \%$ & $60.2 \%$ & $66.3 \%$ & 0.01 \\
\hline \multicolumn{6}{|l|}{ Study Nielsen et al. [60] } \\
\hline Age group (mean) & $79.9(9.0)$ & $77.9(13.5)$ & $83.9(8.2)$ & $71.0(12.6)$ & 0.09 \\
\hline Male & $46.3 \%$ & $46.8 \%$ & $39.4 \%$ & $59.6 \%$ & 0.03 \\
\hline CHA2DS2-VASC (mean) & $3.8(1.5)$ & $3.6(1.8)$ & $4.3(1.5)$ & $3.0(1.7)$ & 0.04 \\
\hline HAS-BLED (mean) & $2.7(1.0)$ & $2.5(1.2)$ & $2.8(1.1)$ & $2.4(1.2)$ & 0.06 \\
\hline $\begin{array}{l}\text { Aspirin or non-steroidal } \\
\text { anti-inflammatory drugs }\end{array}$ & $74.8 \%$ & $66.2 \%$ & $66.7 \%$ & $70.2 \%$ & 0.03 \\
\hline \multicolumn{6}{|l|}{ Study Noseworthy et al. [61] } \\
\hline Age group (median) & $71(62-78)$ & $73(65-81)$ & $73(65-81)$ & NA & - \\
\hline Male & $58.9 \%$ & $54.4 \%$ & $54.1 \%$ & NA & - \\
\hline CHA2DS2-VASC (median) & $4(2-5)$ & $4(3-5)$ & $4(3-5)$ & NA & - \\
\hline HAS-BLED (median) & $2(1-3)$ & $2(2-3)$ & $2(2-3)$ & NA & - \\
\hline $\begin{array}{l}\text { Antiplatelet or non-steroidal } \\
\text { anti-inflammatory drugs }\end{array}$ & $11.1 \%$ & $11.7 \%$ & $12.2 \%$ & NA & - \\
\hline \multicolumn{6}{|l|}{ Study Vinogradova et al. [62] } \\
\hline Age group (mean) & $74.5(10.7)$ & $75.8(10.8)$ & $76.5(10.9)$ & $74.8(10.4)$ & - \\
\hline Male & $59.5 \%$ & $54.3 \%$ & $53.4 \%$ & $55.6 \%$ & - \\
\hline CHA2DS2-VASC & - & - & - & - & - \\
\hline HAS-BLED & - & - & - & - & - \\
\hline $\begin{array}{l}\text { Antiplatelet or non-steroidal } \\
\text { anti-inflammatory drugs }\end{array}$ & $39.6 \%$ & $32.3 \%$ & $31.3 \%$ & $41.8 \%$ & - \\
\hline
\end{tabular}


Table 4. Cont.

\begin{tabular}{cccccc}
\hline & Dabigatran & Rivaroxaban & Apixaban & Warfarin & $\begin{array}{c}\text { Maximum } \\
\text { SMD }\end{array}$ \\
\hline Study Yao et al. [63] & $70(62-78)$ & $72(64-80)$ & $73(66-81)$ & $73(66-81)$ & - \\
\hline Age group (median) & $61.3 \%$ & $57.8 \%$ & $53.1 \%$ & $53.2 \%$ & - \\
\hline Male & $3(2-5)$ & $4(2-5)$ & $4(3-5)$ & $4(3-5)$ & - \\
\hline CHA2DS2-VASC (median) & $2(1-3)$ & $2(2-3)$ & $2(2-3)$ & $2(2-3)$ & - \\
\hline HAS-BLED (median) & $10.3 \%$ & $11.6 \%$ & $12.1 \%$ & $12.5 \%$ & - \\
\hline $\begin{array}{c}\text { Antiplatelet or non-steroidal } \\
\text { anti-inflammatory drugs }\end{array}$ & & & & & \\
\hline
\end{tabular}

SMD-standardized mean difference; CHA2DS2-VASC score-assigns points for the presence of congestive heart failure, hypertension, age 65-74 years and age $\geq 75$ years, diabetes mellitus, stroke or transient ischemic attack, vascular disease, and female sex; HAS-BLED score-assigns points for the presence of hypertension, abnormal renal or liver function, stroke, bleeding history, labile INR, age $\geq 65$ years, and antiplatelet drug or alcohol use; NA-not applicable.

From all of the eleven studies, five of them evaluated the gastrointestinal bleeding risk by comparing each NOAC (dabigatran, rivaroxaban and apixaban) with warfarin, three by direct pairwise comparison of different NOACs and the other three by comparing each NOAC with warfarin and with each other NOAC.

\subsection{Gastrointestinal Bleeding Risk Comparing Each NOAC with Warfarin}

When comparing NOACs with warfarin, we found that in most studies the risk of major gastrointestinal bleeding, which accounted for more than $80 \%$ of major extracranial bleeding, was increased with dabigatran and rivaroxaban and decreased with apixaban, compared with warfarin. The lowest risk of gastrointestinal bleeding was with apixaban compared with warfarin (HR for gastrointestinal bleeding for apixaban ranged between 0.45 (95\% CI 0.34 to 0.59 ) and 1.13 (95\% CI 0.79 to 1.63)). Dabigatran was associated with lower or no significant difference in the risk of gastrointestinal bleeding (HR for dabigatran ranged between 0.58 (95\% CI 0.47 to 0.71 ) and 1.43 (95\% CI 1.07 to 1.90 )) compared to warfarin use. Rivaroxaban had similar or even higher risk of gastrointestinal bleeding compared with warfarin (HR for rivaroxaban ranged between 1.00 (95\% CI 0.87 to 1.16) and 1.38 (95\% CI 1.12 to 1.54$)$ ) (Table 5).

Table 5. Adjusted hazard ratios with $95 \%$ confidence intervals from comparisons of each NOAC versus warfarin for major gastrointestinal bleeding.

\begin{tabular}{cccc}
\hline Study & Dabigatran vs. Warfarin & Rivaroxaban vs. Warfarin & Apixaban vs. Warfarin \\
\hline Forslund et al. [56] & $1.43(1.07-1.9)$ & $1.28(0.90-1.80)$ & $1.13(0.79-1.63)$ \\
Larsen et al. [59] & $0.58(0.47-0.71)$ & $1.06(0.91-1.23)$ & $0.61(0.49-0.75)$ \\
Nielsen et al. [60] & $0.87(0.75-1.01)$ & $1.17(0.94-1.45)$ & $1.04(0.76-1.43)$ \\
Vinogradova et al. [62] & $1.08(0.83-1.41)$ & $1.21(1.01-1.45)$ & $0.76(0.58-0.99)$ \\
Yao et al. [63] & $0.79(0.67-0.94)$ & $1.04(0.90-1.20)$ & $0.45(0.34-0.59)$ \\
Graham et al. [57] & $1.04(0.9-1.21)$ & $1.38(1.12-1.54)$ & $0.51(0.42-0.61)$ \\
Adeboyeje et al. [55] & $1.17(1.04-1.32)$ & $1.00(0.87-1.16)$ & $0.82(0.63-1.06)$ \\
Hernandez et al. [58] & $0.95(0.75-1.19)$ & $1.35(1.20-1.52)$ & $0.72(0.57-0.90)$ \\
\hline
\end{tabular}

It is surprising that the ranges of hazard ratios are so variable between the included studies, especially when comparing gastrointestinal bleeding risk of apixaban vs. warfarin (the ranges being between 0.45 to 1.13). The higher risk for gastrointestinal bleeding of each NOAC versus warfarin encountered during the first year in the study published by Forslund et al. [56] might be related to depletion of susceptibles, the imposibility of quantifying some factors, such as biological age or severity of the co-morbidities, and also the non-excluding design of the study, which included all new users of 
oral anticoagulants from primary health care, hospital based in- and outpatient care or specialized ambulatory care. Subgroup analyses highlighted similar outcomes with warfarin and NOAC treatment in high-risk patients aged 80 and above, and in patients with prior severe bleeds.

\subsection{Gastrointestinal Bleeding Risk by Direct Pairwise Comparison of Different NOACs}

Gastrointestinal bleeding occurred in all of the studies more frequently in patients given rivaroxaban than dabigatran (HR for rivaroxaban ranged between 1.15 (95\% CI 0.99 to 1.36) and 1.35 (95\% CI 0.91 to 2.00)). Apixaban was associated with a lower risk of GI bleeding than dabigatran (HR ranged between 0.39 (95\% CI 0.27 to 0.58 ) and 0.95 (95\% CI 0.65 to 1.18 )) or rivaroxaban (HR ranged between 0.33 (95\% CI 0.22 to 0.49 ) and 0.82 (95\% CI 0.62 to 1.08)). We found that in all of the studies, apixaban had the most favorable GI safety profile and rivaroxaban the least favorable (Table 6).

Table 6. Adjusted hazard ratios with $95 \%$ confidence intervals from direct pairwise comparison of different NOACs for major gastrointestinal bleeding.

\begin{tabular}{cccc}
\hline Study & Rivaroxaban vs. Dabigatran & Apixaban vs. Dabigatran & Apixaban vs. Rivaroxaban \\
\hline Andersson et al. [30] & $1.35(0.91-2.00)$ & $0.94(0.62-1.41)$ & $0.88(0.64-1.22)$ \\
Abraham et al. [54] & $1.20(1.00-1.45)$ & $0.39(0.27-0.58)$ & $0.33(0.22-0.49)$ \\
Noseworthy et al. [61] & $1.30(1.10-1.53)$ & $0.50(0.36-0.70)$ & $0.39(0.28-0.54)$ \\
Graham et al. [57] & $1.32(1.21-1.45)$ & $0.56(0.32-0.74)$ & $0.38(0.27-0.59)$ \\
Adeboyeje et al. [55] & $1.15(0.99-1.36)$ & $0.95(0.65-1.18)$ & $0.82(0.62-1.08)$ \\
Hernandez et al. [58] & $1.25(1.06-1.39)$ & $0.76(0.56-1.03)$ & $0.53(0.42-0.68)$ \\
\hline
\end{tabular}

\section{Discussion}

This study involves all available evidence from high-quality RWSs that were not supported by pharmaceutical companies for a comprehensive and rigorous analysis on the GIB risk for NOACs. The results of our study confirm a low or a similar risk for major GIB between patients receiving apixaban or dabigatran compared with warfarin, and apixaban appears to be associated with the lowest risk of GIB. In most of the studies included in our research, apixaban was associated with a significantly lower risk of gastrointestinal bleeding compared with warfarin. In a direct comparison of the gastrointestinal safety of the NOACs, apixaban appears to be associated with lower risk of gastrointestinal bleeding, whereas rivaroxaban is associated with higher risk of major gastrointestinal bleeding. The once-daily dosing of rivaroxaban and twice-daily administration of dabigatran and apixaban might also explain the higher risk of major bleeding in rivaroxaban, given its higher peak in plasma concentrations [65-68].

The possibility of a different bleeding site according to the type of molecule was discussed in only one study from the selected articles. Thus, in the study published by Vinogradova et al., it was observed that rivaroxaban was associated with higher risk compared with apixaban of a gastrointestinal and upper gastrointestinal bleed, in patients with atrial fibrillation. In those without atrial fibrillation, apixaban was associated with a lower risk of a gastrointestinal and upper gastrointestinal bleed compared with warfarin. Dabigatran and rivaroxaban were associated with a higher risk of a gastrointestinal bleed compared with apixaban, and rivaroxaban was also associated with a higher risk of an upper gastrointestinal bleed [62].

Up to now, there have been systematic reviews and metaanalyses conducted to assess the gastrointestinal risk of NOACs, but from our knowledge, there is no review that has included only real world studies that were not supported by pharmaceutical companies.

Among different NOACs, it is difficult to conclude which drug has the lowest gastrointestinal bleeding risk as there are no direct head-to-head comparisons in randomized clinical trials (RCTs) and because patient characteristics are different across studies. Up to now, several systematic reviews and metaanalyses have been conducted to assess the GIB risk of NOACs in randomized clinical trials. One of the earliest meta-analyses [69], which included 17 RCTs with a total of 75,081 patients who received either a NOAC or standard care (either low-molecular-weight heparin, vitamin K antagonist, 
antiplatelet therapy or placebo), demonstrated a $1.5 \%$ GIB event, with $89 \%$ being major GIB (defined as GIB leading to a decrease in hemoglobin $\geq 2 \mathrm{~g} / \mathrm{dl}$ within $24 \mathrm{~h}$, a transfusion of $\geq 2$ units of packed red cells, necessitating intervention including surgery, or fatal bleeding). Holster et al. also demonstrated that there was an increased risk of GIB among NOAC users compared with standard care and that among different NOACs, dabigatran and rivaroxaban were associated with a higher risk of GIB (OR 1.58 and 1.48, respectively) [69].

In a recent systematic review and meta-analysis of data from randomized controlled trials and real-world studies, Gu et al. [70] evaluated the risk of major gastrointestinal bleeding in patients with NOACs compared with conventional treatment and confirmed that there is no significant difference between these two groups. They analyzed data from 43 randomized controlled trials (183,752 patients) and 41 real-world studies $(1,879,428$ patients). The pooled major rates of GIB for patients on NOACs $(1.19 \%)$ vs conventional treatment $(0.92 \%)$ did not differ significantly (HR from real-world studies, 1.02; 95\% CI, 0.94-1.10; P interaction = 0.52). Rivaroxaban, but not other NOACs, was associated with an increased risk of major GIB (HR from real-world studies, 1.14; 95\% CI, 1.04-1.23; P interaction = 0.06). Rivaroxaban users had a 39\% increase in risk for major GIB. Analyses of subgroups, such as patients with different indications, dosage, or follow-up time, did not significantly affect results.

In another systematic review and meta-analysis, Li et al. [71] summarized the evidence of observational studies for direct comparative safety amongst NOACs in patients with atrial fibrillation. They included fifteen studies for qualitative synthesis and twelve studies for meta-analyses. Rivaroxaban was associated with a significantly higher risk of major bleeding, in comparison with apixaban (HR = 1.71, 95\% CI 1.51-1.94; evidence quality: low). Compared with dabigatran, apixaban was associated with lower risk of major bleeding ( $\mathrm{HR}=0.80,95 \% \mathrm{CI} 0.68-0.95$; evidence quality: low). Apixaban was associated with a lower risk of major bleeding when compared with dabigatran or rivaroxaban, so it was found to have the most favorable safety profile amongst the three NOACs.

The major strength of this study was to reassess the risk for major GIB of NOACs by comparing the results from high-quality RWSs that were not supported by pharmaceutical companies. Certainly there are inherent limitations in this study. First, we included only 11 studies that met the criteria, which may reduce the statistical power. Second, all included studies reported major GIB events according to the International Society on Thrombosis and Hemostasis criteria, but it is not possible to evaluate whether small variations may have an impact on the results obtained. Another weakness of our study is that we did not perform a meta-analysis and, consequently, there are not any pooled estimates of hazard or risk ratios. In addition, we did not have the resources to review non-English articles, but we are confident that this study covered the majority of high-quality RWSs identified in a comprehensive search of broad databases.

\section{Conclusions}

The results of our study from real-world studies confirm a low or a similar risk for major gastrointestinal bleeding between patients receiving NOACs compared with conventional treatment with warfarin. In a direct comparison of the gastrointestinal safety of the NOACs, apixaban appears to be associated with a lower risk of gastrointestinal bleeding, whereas rivaroxaban is associated with a higher risk of major gastrointestinal bleeding. Our findings may provide some decision-making support for physicians regarding their selection of oral anticoagulant treatment based on risk for major gastrointestinal bleeding in patients with AF.

Author Contributions: Conceptualization, L.A. and C.S.; methodology, L.A. and R.S.; validation, L.A., R.S., A.T., I.M.Z. and C.S.; writing—original draft preparation, L.A.; writing—review and editing, L.A., R.S., A.T., I.M.Z. and C.S. All authors have read and agreed to the published version of the manuscript.

Funding: This research received no external funding.

Conflicts of Interest: The authors declare no conflict of interest. 


\section{References}

1. Ogilvie, I.M.; Newton, N.; Welner, S.A.; Cowell, W.; Lip, G.Y. Underuse of oral anticoagulants in atrial fibrillation: A systematic review. Am. J. Med. 2010, 123, 638-645. [CrossRef]

2. Lip, G.Y.; Pan, X.; Kamble, S. Major bleeding risk among non-valvular atrial fibrillation patients initiated on apixaban, dabigatran, rivaroxaban or warfarin: A "real-world" observational study in the United States. Int. J. Clin. Pract. 2016, 70, 752-763. [CrossRef]

3. Maeng, M.; Steg, P.G.; Bhatt, D.L.; Hohnloser, S.H.; Nordaby, M.; Miede, C.; Kimura, T.; Lip, G.Y.; Oldgren, J.; Jurriën, M.; et al. Dabigatran dual therapy versus warfarin triple therapy post-PCI in patients with atrial fibrillation and diabetes. JACC Cardiovasc. Interv. 2019, 12, 2346-2355. [CrossRef]

4. Alak, A.; Hohnloser, S.H.; Fräßdorf, M.; Reilly, P.; Ezekowitz, M.; Healey, J.S.; Brueckmann, M.; Yusuf, S.; Connolly, S.J. Reasons for hospitalization and risk of mortality in patients with atrial fibrillation treated with dabigatran or warfarin in the Randomized Evaluation of Long-term Anticoagulation Therapy (RE-LY) trial. Europace 2019, 21, 1023-1030. [CrossRef]

5. Lanas, A. International consensus guidelines for nonvariceal gastrointestinal bleeding: A step forward. Ann. Intern. Med. 2019, 171, 853-854. [CrossRef]

6. Kjerpeseth, L.J.; Selmer, R.; Ariansen, I.; Karlstad, Ø.; Ellekjaer, H.; Skovlund, E. Comparative effectiveness of warfarin, dabigatran, rivaroxaban and apixaban in non-valvular atrial fibrillation: A nationwide pharmacoepidemiological study. PLoS ONE 2019, 14, e0221500. [CrossRef]

7. Barkun, A.N.; Almadi, M.; Kuipers, E.J.; Laine, L.; Sung, J.; Tse, F.; Leontiadis, G.I.; Abraham, N.S.; Calvet, X.; Chan, F.K.; et al. Management of nonvariceal upper gastrointestinal bleeding: Guideline recommendations from the international consensus group. Ann. Intern. Med. 2019, 171, 805-822. [CrossRef]

8. Jansson, M.; Själander, S.; Sjögren, V.; Renlund, H.; Norrving, B.; Själander, A. Direct comparisons of effectiveness and safety of treatment with Apixaban, Dabigatran and Rivaroxaban in atrial fibrillation. Thromb. Res. 2020, 185, 135-141. [CrossRef] [PubMed]

9. Inoue, H.; Uchiyama, S.; Atarashi, H.; Okumura, K.; Koretsune, Y.; Yasaka, M.; Yamashita, T.; Taniguchi, A.; Fukaya, T. J-dabigation surveillance investigators. Effectiveness and safety of long-term dabigatran among patients with non-valvular atrial fibrillation in clinical practice: J-dabigatran surveillance. J. Cardiol. 2019, 73, 507-514. [CrossRef] [PubMed]

10. Sostres, C.; Marcén, B.; Laredo, V.; Alfaro, E.; Ruiz, L.; Camo, P.; Carrera-Lasfuentes, P.; Lanas, Á. Risk of rebleeding, vascular events and death after gastrointestinal bleeding in anticoagulant and/or antiplatelet users. Aliment. Pharmacol. Ther. 2019, 50, 919-929. [CrossRef] [PubMed]

11. Briere, J.B.; Bowrin, K.; Coleman, C.; Fauchier, L.; Levy, P.; Folkerts, K.; Toumi, M.; Taieb, V.; Millier, A.; $\mathrm{Wu}, \mathrm{O}$; ; et al. Real-world clinical evidence on rivaroxaban, dabigatran, and apixaban compared with vitamin $\mathrm{K}$ antagonists in patients with nonvalvular atrial fibrillation: A systematic literature review. Expert Rev. Pharmacoecon. Outcomes Res. 2019, 19, 27-36. [CrossRef] [PubMed]

12. Rutherford, O.C.; Jonasson, C.; Ghanima, W.; Söderdahl, F.; Halvorsen, S. Comparison of dabigatran, rivaroxaban and apixaban for effectiveness and safety in atrial fibrillation; a nationwide cohort study. Eur. Heart J. Cardiovasc. Pharmacother. 2020, 6, 75-85. [CrossRef] [PubMed]

13. Lanas-Gimeno, A.; Lanas, A. Risk of gastrointestinal bleeding during anticoagulant treatment. Expert Opin. Drug Saf. 2017, 16, 673-685. [CrossRef] [PubMed]

14. Stam-Slob, M.C.; Connolly, S.J.; van der Graaf, Y.; van der Leeuw, J.; Dorresteijn, J.A.; Eikelboom, J.W.; Peters, R.J.; Alings, M.; Visseren, F.L. Individual treatment effect estimation of 2 doses of Dabigatran on stroke and major bleeding in atrial fibrillation: Results from the RE-LY trial. Circulation 2019, 139, 2846-2856. [CrossRef]

15. Hohnloser, S.H.; Fudim, M.; Alexander, J.H.; Wojdyla, D.M.; Ezekowitz, J.A.; Hanna, M.; Atar, D.; Hijazi, Z.; Bahit, M.C.; Al-Khatib, S.M.; et al. Efficacy and safety of apixaban versus warfarin in patients with atrial fibrillation and extremes in body weight: Insights from the ARISTOTLE trial. Circulation 2019, 139, 2292-2300. [CrossRef]

16. Martinez, B.K.; Sood, N.A.; Bunz, T.J.; Coleman, C.I. Effectiveness and safety of apixaban, dabigatran, and rivaroxaban versus warfarin in frail patients with nonvalvular atrial fibrillation. J. Am. Heart Assoc. 2018, 7, e008643. [CrossRef] 
17. Villines, T.C.; Ahmad, A.; Petrini, M.; Tang, W.; Evans, A.; Rush, T.; Thompson, D.; Oh, K.; Schwartzman, E. Comparative safety and effectiveness of dabigatran vs. rivaroxaban and apixaban in patients with non-valvular atrial fibrillation: A retrospective study from a large healthcare system. Eur. Heart J. Cardiovasc. Pharmacother. 2019, 5, 80-90. [CrossRef]

18. Lanas, Á.; Carrera-Lasfuentes, P.; Arguedas, Y.; García, S.; Bujanda, L.; Calvet, X.; Ponce, J.; Perez-Aísa, Á.; Castro, M.; Muñoz, M.; et al. Risk of upper and lower gastrointestinal bleeding in patients taking nonsteroidal anti-inflammatory drugs, antiplatelet agents, or anticoagulants. Clin. Gastroenterol. Hepatol. 2015, 13, 906-912. [CrossRef]

19. Desai, J.; Kolb, J.M.; Weitz, J.I.; Aisenberg, J. Gastrointestinal bleeding with the new oral anticoagulants-defining the issues and the management strategies. Thromb. Haemost. 2013, 110, $205-212$. [CrossRef]

20. Cheung, K.S.; Leung, W.K. Gastrointestinal bleeding in patients on novel oral anticoagulants: Risk, prevention and management. World J. Gastroenterol. 2017, 23, 1954-1963. [CrossRef]

21. Cha, M.J.; Choi, E.K.; Han, K.D.; Lee, S.-R.; Lim, W.-H.; Oh, S.; Lip, G.Y. Effectiveness and safety of non-vitamin $\mathrm{K}$ antagonist oral anticoagulants in Asian patients with atrial fibrillation. Stroke 2017, 48, 3040-3048. [CrossRef] [PubMed]

22. Antza, C.; Doundoulakis, I.; Akrivos, E.; Economou, F.; Vazakidis, P.; Haidich, A.B.; Kotsis, V. Non-vitamin K oral anticoagulants in nonvalvular atrial fibrillation: A network meta-analysis. Scand. Cardiovasc. J. 2019, 53, 48-54. [CrossRef] [PubMed]

23. Chan, Y.H.; Lee, H.F.; See, L.C.; Tu, H.T.; Chao, T.F.; Yeh, Y.H.; Wu, L.S.; Kuo, C.T.; Chang, S.H.; Lip, G.Y.; et al. Effectiveness and safety of four direct oral anticoagulants in Asian patients with nonvalvular atrial fibrillation. Chest 2019, 156, 529-543. [CrossRef] [PubMed]

24. Kolb, J.M.; Flack, K.F.; Chatterjee-Murphy, P. The locations and mucosal lesions responsible for major gastrointestinal bleeding in patients on warfarin or dabigatran. Dig. Dis. Sci. 2018, 63, 1878-1889. [CrossRef]

25. Brodie, M.M.; Newman, J.C.; Smith, T.; Rockey, D.C. Severity of gastrointestinal bleeding in patients treated with direct-acting oral anticoagulants. Am. J. Med. 2018, 131, e9-e573. [CrossRef]

26. Paquette, M.; França, L.R.; Teutsch, C.; Diener, H.C.; Lu, S.; Dubner, S.J.; Ma, C.S.; Rothman, K.J.; Zint, K.; Halperin, J.L.; et al. Dabigatran persistence and outcomes following discontinuation in atrial fibrillation patients from the GLORIA-AF registry. Am. J. Cardiol. 2020, 125, 383-391. [CrossRef]

27. Tsuda, K.; Natori, T.; Shimizu, M.; Ishigaku, Y.; Oi, K.; Narumi, S.; Kamada, A.; Yoshida, M.; Oura, K.; Maeda, T.; et al. Novel antithrombotic effects of dabigatran in patients with non-valvular atrial fibrillation. Thromb. Res. 2020, 189, 1-4. [CrossRef]

28. Fauchier, L.; Blin, P.; Sacher, F.; Dureau-Pournin, C.; Bernard, M.A.; Lassalle, R.; Droz-Perroteau, C.; Dallongeville, J.; Moore, N. Reduced dose of rivaroxaban and dabigatran vs. vitamin K antagonists in very elderly patients with atrial fibrillation in a nationwide cohort study. Europace 2020, 22, 205-215. [CrossRef]

29. Cangemi, D.J.; Krill, T.; Weideman, R.; Cipher, D.J.; Spechler, S.J.; Feagins, L.A. A comparison of the rate of gastrointestinal bleeding in patients taking non-vitamin $\mathrm{K}$ antagonist oral anticoagulants or Warfarin. Am. J. Gastroenterol. 2017, 112, 734-739. [CrossRef]

30. Andersson, N.W.; Svanstrom, H.; Lund, M.; Pasternak, B.; Melbye, M. Comparative effectiveness and safety of apixaban, dabigatran, and rivaroxaban in patients with non-valvular atrial fibrillation. Int. J. Cardiol. 2018, 268, 113-119. [CrossRef]

31. Nissan, R.; Spectre, G.; Hershkovitz, A.; Green, H.; Shimony, S.; Cooper, L.; Nakav, S.; Shochat, T.; Grossman, A.; Fuchs, S.; et al. Apixaban levels in octogenarian patients with non-valvular atrial fibrillation. Drugs Aging 2019, 36, 165-177. [CrossRef] [PubMed]

32. Alexander, K.P.; Brouwer, M.A.; Mulder, H.; Vinereanu, D.; Lopes, R.D.; Proietti, M.; Al-Khatib, S.M.; Hijazi, Z.; Halvorsen, S.; Hylek, E.M.; et al. Outcomes of apixaban versus warfarin in patients with atrial fibrillation and multi-morbidity: Insights from the ARISTOTLE trial. Am. Heart J. 2019, 208, $123-131$. [CrossRef] [PubMed]

33. Schneeweiss, S. Effectiveness and safety of Apixaban versus Rivaroxaban for patients with atrial fibrillation. Ann. Intern. Med. 2020, 172, 1-16. 
34. Fralick, M.; Colacci, M.; Schneeweiss, S.; Huybrechts, K.F.; Lin, K.J.; Gagne, J.J. Effectiveness and safety of apixaban compared with rivaroxaban for patients with atrial fibrillation in routine practice: A cohort study. Ann. Intern. Med. 2020, 172, 463. [CrossRef] [PubMed]

35. Cîmpan, P.L.; Chira, R.I.; Mocan, M.; Anton, F.P.; Farcaș, A.D. Oral anticoagulant therapy—When art meets science. J. Clin. Med. 2019, 8, 1747. [CrossRef] [PubMed]

36. Lip, G.Y.; Khan, A.A.; Olshansky, B. Short-term outcomes of apixaban versus warfarin in patients with atrial fibrillation: Is body weight an important consideration? Circulation 2019, 139, 2301-2303. [CrossRef]

37. Verdecchia, P.; D'Onofrio, A.; Russo, V.; Fedele, F.; Adamo, F.; Benedetti, G.; Ferrante, F.; Lodigiani, C.; Paciullo, F.; Aita, A.; et al. Persistence on apixaban in atrial fibrillation patients: A retrospective multicentre study. J. Cardiovasc. Med. 2019, 20, 66-73. [CrossRef]

38. Wanat, M.A.; Wang, X.; Paranjpe, R.; Chen, H.; Johnson, M.L.; Fleming, M.L.; Abughosh, S.M. Warfarin vs. apixaban in nonvalvular atrial fibrillation, and analysis by concomitant antiarrhythmic medication use: A national retrospective study. Res. Pract. Thromb. Haemost. 2019, 3, 674-683. [CrossRef]

39. Ramagopalan, S.V.; Sicras-Mainar, A.; Polanco-Sanchez, C.; Carroll, R.; de Bobadilla, J.F. Patient characteristics and stroke and bleeding events in nonvalvular atrial fibrillation patients treated with apixaban and vitamin K antagonists: A Spanish real-world study. J. Comp. Eff. Res. 2019, 8, 1201-1212. [CrossRef]

40. Washam, J.B.; Hohnloser, S.H.; Lopes, R.D.; Wojdyla, D.M.; Vinereanu, D.; Alexander, J.H.; Gersh, B.J.; Hanna, M.; Horowitz, J.; Hylek, E.M.; et al. Interacting medication use and the treatment effects of apixaban versus warfarin: Results from the ARISTOTLE Trial. J. Thromb. Thrombolysis 2019, 47, 345-352. [CrossRef]

41. Abraham, N.S.; Singh, S.; Alexander, G.C. Comparative risk of gastrointestinal bleeding with dabigatran, rivaroxaban, and warfarin: Population based cohort study. BMJ 2015, 350, h1857. [CrossRef] [PubMed]

42. Camm, A.J.; Amarenco, P.; Haas, S.; Hess, S.; Kirchhof, P.; Lambelet, M.; Bach, M.; Turpie, A.G. Real-world vs. randomized trial outcomes in similar populations of rivaroxaban-treated patients with non-valvular atrial fibrillation in ROCKET AF and XANTUS. Europace 2019, 21, 421-427. [CrossRef] [PubMed]

43. Beyer-Westendorf, J.; Camm, A.J.; Fox, K.A.; Le Heuzey, J.Y.; Haas, S.; Turpie, A.G.; Virdone, S.; Kakkar, A.K.; RIVER Registry Investigators. International longitudinal registry of patients with atrial fibrillation and treated with rivaroxaban: RIVaroxaban Evaluation in Real life setting (RIVER). Thromb. J. 2019, $17,7$. [CrossRef]

44. Lin, S.Y.; Kuo, C.H.; Yeh, S.J.; Tsai, L.K.; Liu, Y.B.; Huang, C.F.; Tang, S.C.; Jeng, J.S. Real-world rivaroxaban and apixaban levels in Asian patients with atrial fibrillation. Clin. Pharmacol. Ther. 2020, 107, 278-286. [CrossRef]

45. Sakuma, I.; Uchiyama, S.; Atarashi, H.; Inoue, H.; Kitazono, T.; Yamashita, T.; Shimizu, W.; Ikeda, T.; Kamouchi, M.; Kaikita, K.; et al. Clinical risk factors of stroke and major bleeding in patients with non-valvular atrial fibrillation under rivaroxaban: The EXPAND Study sub-analysis. Heart Vessel. 2019, 34, 1839-1851. [CrossRef] [PubMed]

46. Reilly, P.A.; Lehr, T.; Haertter, S.; Connolly, S.; Yusuf, S.; Eikelboom, J.; Ezekowitz, M.D.; Nehmiz, G.; Wang, S.; Wallentin, L.; et al. The effect of dabigatran plasma concentrations and patient characteristics on the frequency of ischemic stroke and major bleeding in atrial fibrillation patients: The RE-LY Trial (Randomized Evaluation of Long-Term Anticoagulation Therapy). J. Am. Coll. Cardiol. 2014, 63, 321-328. [CrossRef]

47. Chang, H.Y.; Zhou, M.; Tang, W.; Alexander, G.C.; Singh, S. Risk of gastrointestinal bleeding associated with oral anticoagulants: Population based retrospective cohort study. BMJ 2015, 350, h1585. [CrossRef]

48. Sung, J.J.; Chiu, P.W.; Chan, F.K.; Lau, J.Y.; Goh, K.L.; Ho, L.H.; Jung, H.Y.; Sollano, J.D.; Gotoda, T.; Reddy, N.; et al. Asia-Pacific working group consensus on non-variceal upper gastrointestinal bleeding: An update 2018. Gut 2018, 67, 1757-1768. [CrossRef]

49. Lansas, A.; Dumonceau, J.M.; Hunt, R.H.; Fujishiro, M.; Scheiman, J.M.; Gralnek, I.M.; Campbell, H.E.; Rostom, A.; Villanueva, C.; Sung, J.J.Y.; et al. Non-variceal upper gastrointestinal bleeding. Nat. Rev. Dis. Prim. 2018, 4, 18020. [CrossRef]

50. Zhou, Y.; Guo, Y.; Liu, D.; Feng, H.; Liu, J. Restarting of anticoagulation in patients with atrial fibrillation after major bleeding: A meta-analysis. J. Clin. Pharm. Ther. 2020. [CrossRef]

51. Contaldo, A.; Losurdo, G.; Albano, F.; Iannone, A.; Michele, B.; Ierardi, E.; Di Leo, A.; Principi, M. The spectrum of small intestinal lesions in patients with unexplained iron deficiency anemia detected by video capsule endoscopy. Medicina 2019, 55, 59. [CrossRef] [PubMed] 
52. Sjogren, V.; Bystrom, B.; Renlund, H. Non-vitamin K oral anticoagulants are non-inferior for stroke prevention but cause fewer major bleedings than well-managed warfarin: A retrospective register study. PLoS ONE 2017, 12, e0181000. [CrossRef] [PubMed]

53. Friberg, L.; Oldgren, J. Efficacy and safety of non-vitamin $\mathrm{K}$ antagonist oral anticoagulants compared with warfarin in patients with atrial fibrillation. Open Heart 2017, 4, e000682. [CrossRef] [PubMed]

54. Abraham, N.S.; Noseworthy, P.A.; Yao, X. Gastrointestinal safety of direct oral anticoagulants: A large population-based study. Gastroenterology 2017, 152, 1014-1022. [CrossRef] [PubMed]

55. Adeboyeje, G.; Sylwestrzak, G.; Barron, J.J. Major bleeding risk during anticoagulation with warfarin, dabigatran, apixaban, or rivaroxaban in patients with nonvalvular atrial fibrillation. J. Manag. Care Spec. Pharm. 2017, 23, 968-978. [CrossRef]

56. Forslund, T.; Wettermark, B.; Andersen, M.; Hjemdahl, P. Stroke and bleeding with non-vitamin K antagonist oral anticoagulant or warfarin treatment in patients with non-valvular atrial fibrillation: A population-based cohort study. Europace 2018, 20, 420-428. [CrossRef]

57. Graham, D.J.; Baro, E.; Zhang, R. Comparative stroke, bleeding, and mortality risks in older Medicare patients treated with oral anticoagulants for nonvalvular atrial fibrillation. Am. J. Med. 2019, 132, 596-604. [CrossRef]

58. Hernandez, I.; Zhang, Y.; Saba, S. Comparison of the effectiveness and safety of apixaban, dabigatran, rivaroxaban, and warfarin in newly diagnosed atrial fibrillation. Am. J. Cardiol. 2017, 120, 1813-1819. [CrossRef]

59. Larsen, T.B.; Skjoth, F.; Nielsen, P.B. Comparative effectiveness and safety of non-vitamin K antagonist oral anticoagulants and warfarin in patients with atrial fibrillation: Propensity weighted nationwide cohort study. BMJ 2016, 353, i3189. [CrossRef]

60. Nielsen, P.B.; Skjoth, F.; Sogaard, M. Effectiveness and safety of reduced dose non-vitamin K antagonist oral anticoagulants and warfarin in patients with atrial fibrillation: Propensity weighted nationwide cohort study. BMJ 2017, 356, j510. [CrossRef]

61. Noseworthy, P.A.; Yao, X.; Abraham, N.S.; Sangaralingham, L.R.; McBane, R.D.; Shah, N.D. Direct comparison of dabigatran, rivaroxaban, and apixaban for effectiveness and safety in nonvalvular atrial fibrillation. CHEST 2016, 150, 1302-1312. [CrossRef] [PubMed]

62. Vinogradova, Y.; Coupland, C.; Hill, T. Risks and benefits of direct oral anticoagulants versus warfarin in a real world setting: Cohort study in primary care. BMJ 2018, 362, k2505. [CrossRef] [PubMed]

63. Yao, X.; Abraham, N.S.; Sangaralingham, L.R. Effectiveness and safety of dabigatran, rivaroxaban, and apixaban versus warfarin in nonvalvular atrial fibrillation. J. Am. Heart Assoc. 2016, 5, e003725. [CrossRef]

64. Schulman, S.; Angeras, U.; Bergqvist, D. Definition of major bleeding in clinical investigations of antihemostatic medicinal products in surgical patients. J. Thromb. Haemost. 2010, 8, 202-204. [CrossRef] [PubMed]

65. Moon, I.; Lee, S.R.; Choi, E.K.; Lee, E.; Jung, J.H.; Han, K.D.; Cha, M.J.; Oh, S.; Lip, G.Y.H. Non-vitamin K antagonist oral anticoagulants in patients with atrial fibrillation and valvular heart disease. J. Clin. Med. 2019, 8, 1624. [CrossRef]

66. Yap, L.B.; Eng, D.T.; Sivalingam, L. A comparison of dabigatran with warfarin for stroke prevention in atrial fibrillation in an Asian population. Clin. Appl. Thromb. Hemost. 2016, 22, 792-797. [CrossRef]

67. Mitchell, A.; Watson, M.C.; Welsh, T.; McGrogan, A. Effectiveness and safety of direct oral anticoagulants versus vitamin $\mathrm{K}$ antagonists for people aged 75 years and over with atrial fibrillation: A systematic review and meta-analyses of observational studies. J. Clin. Med. 2019, 8, 554. [CrossRef]

68. Park, J.; Lee, S.R.; Choi, E.K.; Kwon, S.; Jung, J.H.; Han, K.D.; Cha, M.J.; Ko, S.B.; Oh, S.; Lip, G.Y.H.; et al. Effectiveness and safety of direct oral anticoagulants for secondary prevention in Asians with atrial fibrillation. J. Clin. Med. 2019, 8, 2228. [CrossRef]

69. Holster, I.L.; Valkhoff, V.E.; Kuipers, E.J. New oral anticoagulants increase risk for gastrointestinal bleeding: A systematic review and meta-analysis. Gastroenterology 2013, 145, 105-112. [CrossRef] 
70. Gu, Z.H.; Wei, A.H.; Zhang, C. Risk of major gastrointestinal bleeding with new vs conventional oral anticoagulants: A systematic review and meta-analysis. Clin. Gastroenterol. Hepatol. 2020, 18, 792-799. [CrossRef]

71. Li, G.; Lip, G.Y.H.; Holbrook, A. Direct comparative effectiveness and safety between non-vitamin K antagonist oral anticoagulants for stroke prevention in nonvalvular atrial fibrillation: A systematic review and meta-analysis of observational studies. Eur. J. Epidemiol. 2019, 34, 173-190. [CrossRef] [PubMed] 